\title{
Original
}

\section{Relationship between Lung Lobar Volume Ratio and Deformity of the Orifice of the Right Middle Lobe Bronchus after Right Upper Lobectomy}

\author{
Makoto Nonaka, Mitsutaka Kadokura, Shigeru Yamamoto, \\ Daisuke Kataoka, Tamio KushiHashi ${ }^{*}$, Tadanori Kawada \\ and Toshihiro TAKABA
}

\begin{abstract}
After right upper (RU) lobectomy, a deformity of the orifice of the right middle lobe bronchus (RMLB) may be observed in some cases. In this study, we examined the relationship between the postoperative deformity of the orifice of the RMLB (ORMLB) and the pre- and post-operative right lung lobar volume ratio. Before and after RU lobectomy, the lobar volume ratio was calculated using computed tomography and the ORMLB was measured using bronchofiberscopy $(n=19)$. A preoperative ratio of the volume of the right upper lobe (RUL) to the volume of the right lung was not correlated with the postoperative deformity of the ORMLB. When a postoperative ratio of the volume of the right middle lobe (RML) to the volume of the right middle and lower lobes (RMLL) was less than 30\%, postoperative deformity of the ORMLB was observed in a case who had preoperative semicircular ORMLB, but not in a case who had a round-shaped ORMLB preoperatively. To study the postoperative change in the RML to RMLL ratio, the preoperative RML to RMLL volume ratio was divided by the postoperative volume ratio. The ORMLB was deformed when the ratio was less than $60 \%$, however, there was no deformity if the ratio was greater than $60 \%$, regardless of the preoperative shape of the ORMLB. In conclusion, the preoperative volume ratio of the RUL to the right lung is not useful to predict the postoperative deformity of the ORMLB. Deformation of the ORMLB is not observed when the preoperative ratio of the volume of the RML to the volume of the RMLL is preserved after surgery.
\end{abstract}

Key words: lung lobar volume, bronchofiberscopy, right upper lobectomy, bronchial deformity, right middle lobe

\section{Introduction}

After right upper (RU) lobectomy, the orifice of the right middle lobe bronchus (RMLB), which is oriented in a caudal direction, may be deformed by repositioning of the remaining right middle lobe $(\mathrm{RML})$ and right lower lobe $(\mathrm{RLL})^{1)}$. Previously, Leuven et

First Department of Surgery, Showa University School of Medicine, 1-5-8 Hatanodai, Shinagawa-ku, Tokyo 1428666, Japan.

* Department of Radiology, Showa University School of Medicine. 
al. ${ }^{2)}$ and $\mathrm{we}^{3)}$ reported residual bronchial obstruction after upper lobectomy, but the mechanism is still unknown. This bronchial branching deformity after RU lobectomy is a passive change, which occurs until the dead space of thoracic cage is filled by the remaining lobes. It is not clear whether the postoperative deformity is affected by the volume of the resected right upper lobe (RUL), or whether the bronchial deformity is influenced by the pre- and post-operative right lobar volume ratios. The purpose of this study was to determine how different right lung lobar volume ratios contribute to deformity of the orifice of the RMLB (ORMLB) after RU lobectomy.

\section{Patients and Methods}

Computed tomography (CT) of the chest and bronchofiberscopy (BFS) were performed in 19 patients before and after RU lobectomy. The postoperative CT and BFS were performed after the remaining lobes were fully expanded and pleural effusion was absorbed. All patients had peripheral lung cancer with no bronchial branching deformity before surgery. The diameter of the tumor ranged from $10 \mathrm{~mm}$ to $35 \mathrm{~mm}$ (average $23 \mathrm{~mm}$ ). Obstructive pneumonia due to the tumor was not prominent. Patients with emphysema were excluded from this study. The RUL bronchus was incised at the level of the bifurcation and the stump was closed with absorbable monofilament sutures. The suture line of the stump was placed across the longitudinal axis of the remnant bronchus. Patients who underwent RU lobectomy with wedge resection, sleeve resection, partial resection of the other lobes, or chest wall resection were excluded.

The patients were divided into four groups according to the BFS findings before and after RU lobectomy. In general, the ORMLB is a round or semicircular shape, depending on the relationship between the membranous portion and bronchial cartilage (Fig. 1). We previously defined the preoperative ORMLB as round-shaped if the conjugate-to-transversal axis ratio $(\mathrm{c} / \mathrm{t}$ ratio) of the ORMLB was over $60 \%$, and as semicircular-shaped when the $\mathrm{c} / \mathrm{t}$ ratio was under $60 \%{ }^{4)}$. In the preoperatively round group, when the postoperative $\mathrm{c} / \mathrm{t}$ ratio was over $40 \%$, the case was defined as "not deformed" and the case was categorized in the $R R$ group. If the postoperative $c / t$ ratio was under $40 \%$ in a preoperatively

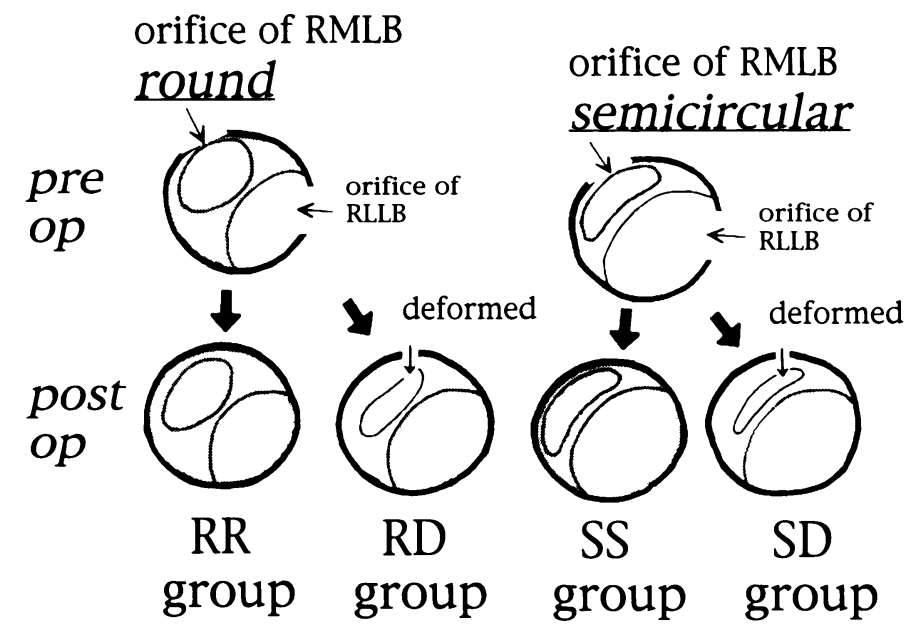

Fig. 1. Group 
round-shaped group, the case was defined as "deformed" and was placed in the RD group (Fig. 1). In the preoperatively semicircular-shaped group, when the postoperative $\mathrm{c} / \mathrm{t}$ ratio was over $40 \%$, the case was defined as "not deformed" and put in the SS group. If the postoperative $\mathrm{c} / \mathrm{t}$ ratio was under $40 \%$ in a preoperatively semicircular-shaped case, they were defined as "deformed" and put in the SD group (Fig. 1).

The lung lobar volume ratios were calculated using chest $\mathrm{CT}^{5-8)}$ before and after RU lobectomy. A full chest CT scan was performed and the area of each tracing was measured. Each area was multiplied by slice thickness and each lobe volume ratio in the right lung was calculated. The preoperative ratio of the volume of the RUL to the volume of the right lung was calculated. The pre- and post-operative ratio of the volume of the RML to the volume of the right middle and lower lobes (RMLL) was also calculated.

The relationship between the lung lobar volume ratio and the deformity of the ORMLB was analyzed. The results were presented as means and the standard error of the mean. The significance of difference between the groups was estimated by analysis of variance and the differences were considered significant when the $\mathrm{p}$ value was less than 0.05 .

\section{Results}

Eight cases were categorized in the RR group, 3 in the RD group, 4 in the SS group, and 4 in the SD group. The ratio of the volume of the RUL to the volume of the right lung was $38 \%$ before RU lobectomy (Table 1). The preoperative volume ratio of the RUL to the right lung was not different among the groups (n.s., Fig. 2, Table 2).

In cases who had preoperative semicircular ORMLB, the relationship between the postoperative deformation of the ORMLB and the postoperative ratios of the volume of the RML to the volume of the RMLL was studied (Fig. 3, SS and SD groups). When the volume ratio of the RML to the RMLL was less than $30 \%$ after RU lobectomy, the ORMLB was deformed postoperatively (SD group). If the ratio of the RML to the RMLL was greater than $30 \%$ after surgery, the ORMLB was not deformed (SS group). However, in cases who had preoperative round ORMLB, the postoperative deformation of the

Table 1. Lobar volume ratios before and after right upper (RU) lobectomy.

\begin{tabular}{lcc}
\hline & Before RU lobectomy & After RU lobectomy \\
\hline RUL & $37.9 \pm 1.6 \%$ & $(-)$ \\
RML & $19.8 \pm 0.9 \%$ & $27.6 \pm 2.9 \%$ \\
RLL & $42.3 \pm 2.0 \%$ & $72.4 \pm 2.9 \%$ \\
\hline
\end{tabular}

RUL, right upper lobe ; RML, right middle lobe ; RLL, right lower lobe. data are presented as the mean \pm the standard error of the mean

Table 2. Lobar volume ratios before right upper lobectomy.

\begin{tabular}{lcccc}
\hline & RR group & RD group & SS group & SD group \\
\hline RUL & $37.5 \pm 3.1 \%$ & $34.3 \pm 0.9 \%$ & $38.2 \pm 3.2 \%$ & $41.0 \pm 3.7 \%$ \\
RML & $20.2 \pm 1.7 \%$ & $19.3 \pm 2.4 \%$ & $18.8 \pm 2.1 \%$ & $20.2 \pm 0.6 \%$ \\
RLL & $42.2 \pm 4.1 \%$ & $46.3 \pm 2.6 \%$ & $43.0 \pm 3.3 \%$ & $38.8 \pm 3.3 \%$ \\
\hline
\end{tabular}

RUL, right upper lobe ; RML, right middle lobe ; RLL, right lower lobe. data are presented as the mean \pm the standard error of the mean 


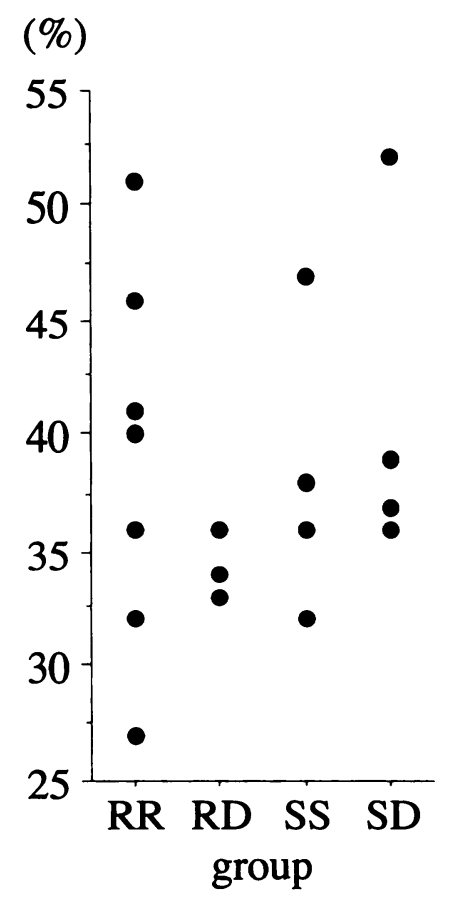

Fig. 2. Ratio of the volume of the right upper lobe to the volume of the right lung before right upper lobectomy in each group.

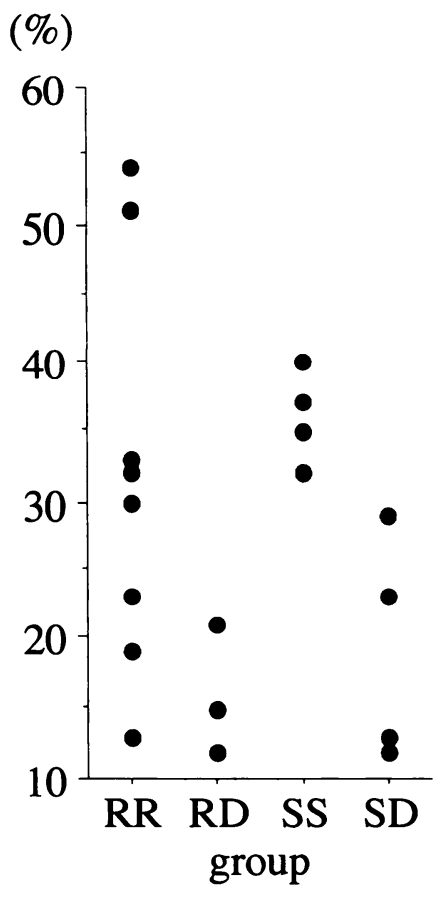

Fig. 3. Ratio of the volume of the right middle lobe (RML) to the volume of the RML and right lower lobe ( RLL) after right upper lobectomy in each group. The ratio was calculated by following formula:

post RML

post $\mathrm{RML}+$ post RLL

ORMLB was not associated with the postoperative volume ratio of the RML to the RMLL (Fig. 3, RR and RD groups).

The ratio of the volume of the RML to the volume of the RMLL was compared before and after RU lobectomy. The ratio of the volume of the RML to the volume of the RMLL after RU lobectomy was divided by the ratio of the volume of the RML to the volume of the RMLL before RU lobectomy (Fig. 4). The ORMLB was deformed (RD and SD groups) when the ratio was less than $60 \%$ regardless of the preoperative shape of the ORMLB, but when the ratio was greater than $60 \%$, the ORMLB was never deformed (RR and SS groups, Fig. 4).

\section{Discussion}

After lobectomy, the dead space is filled by mediastinal shift, elevation of the diaphragm, enlargement and shift of the remaining lobes, and reduction of the thoracic cage ${ }^{9,10)}$. Because the bronchus is passively deformed after lobectomy by the enlargement and shift of the remaining lobes, it has been postulated that preoperative volume ratios of the RUL to the right lung should affect the postoperative bronchial branching deformity. However, in 


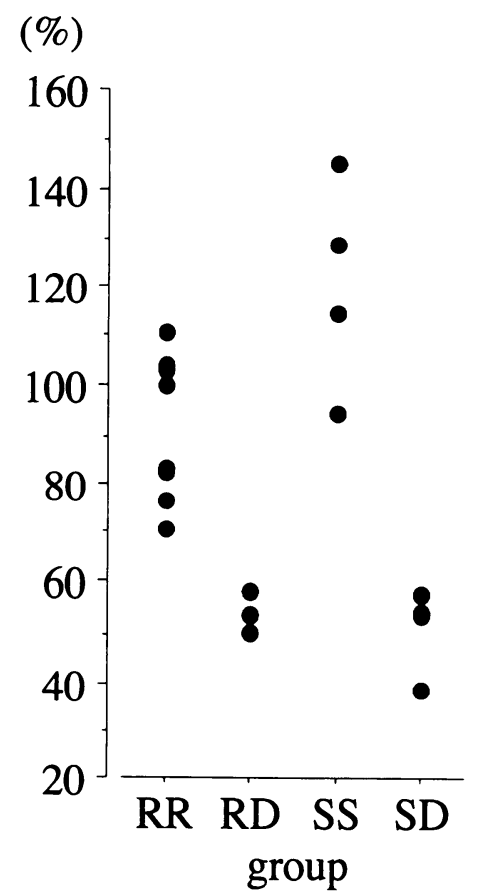

Fig. 4. Ratio of the volume of the right middle lobe (RML) to the volume of the RML and right lower lobe (RLL) after right upper lobectomy divided by the ratio of the volume of the RML to the volume of the RML and RLL before surgery in each group. The ratio was calculated by following formula:

$\frac{\text { post } R M L /(\text { post } R M L+\text { post RLL) }}{\text { pre RML/ (pre RML + pre RLL) }}$

the present study, an unexpected finding was that the deformity of the ORMLB was not correlated with the preoperative ratio of the volume of the RUL to the volume of the right lung (Fig. 2). Therefore, the preoperative RUL volume ratio may not predict the postoperative deformity of the ORMLB.

In this study, postoperative deformity of the preoperatively semicircular ORMLB was observed when the postoperative ratio of the RML to the RMLL was under $30 \%$. In addition, postoperative deformity of the ORMLB was never observed when the postoperative ratio of the RML to the RMLL was over $30 \%$ in preoperatively semicircular ORMLB cases. These results indicate that the postoperative volume ratio of the RML to the RMLL is a good predictor for the deformation of the ORMLB in cases with a preoperative semicircular ORMLB. Meanwhile, postoperative deformity of the preoperatively round ORMLB was not observed in some cases even though the postoperative volume ratio of the RML to the RMLL was extremely low (RR group, Fig. 3). Since the preoperatively round orifice cases have rigid and circular bronchial ORMLB, these cases can tolerate a kink or bend in the ORMLB even if the postoperative RML volume ratio is low. 
It is clear from our study that a postoperative decrease in the ratio of the volume of the RML to the volume of the RMLL is associated with deformity of the ORMLB, regardless of the preoperative shape of the ORMLB (Fig. 4). The bronchial deformity after lobectomy is a passive change that remains until the dead space of the thoracic cage is filled by the remaining lobes ${ }^{11)}$. When stenosis of the ORMLB is occurs during this period, air passage to the RML may decrease, the postoperative $R M L$ volume ratio will reduce gradually, and the postoperative deformity of the ORMLB will be severe. If the ratio of the volume of the RML to the volume of the RMLL after RU lobectomy is divided by a ratio of the volume of the RML to the volume of the RMLL before RU lobectomy, and if physicians notice that the ratio is less than $60 \%$, deformation of the ORMLB should be suspected. To our knowledge, this is the first report to quantify the safety lobar volume ratio associated with the $\mathrm{RU}$ lobectomy in man.

We previously described how different surgical methods for right upper lobectomy can contribute to the postoperative bronchial branching deformity using an animal model ${ }^{12)}$. Based on these results, patients who underwent RU lobectomy with wedge resection, sleeve resection, or partial resection of the other lobes were excluded in the present study.

In conclusion, the preoperative ratio of the volume of the RUL to the volume of the right lung is not useful to predict the postoperative bronchial deformity. When the RLL is overinflated after RU lobectomy, deformity of the ORMLB is observed.

\section{Acknowledgments}

The authors wish to thank the staff of the Photo Center at Showa University for their technical assistance. This work was presented at the 38th World Congress of Surgery of the International Scciety of Surgery, held in Vienna, Austria, August 15-20, 1999.

\section{References}

1) Nonaka M, Kadokura M, Tanio N, Yamamoto S, Kataoka D, Bitoh A, Matsuoka J, Inoue K and Takaba T: Bronchoscopic treatment for retention of bronchial secretions after thoracic and cardiovascular surgery. $J J p n$ Soc Bronchol 19 : 25-28 (1997) (in Japanese)

2) Leuven MV, Clayman JA and Snow N : Bronchial obstruction after upper lobectomy: kinked bronchus treated by stenting. Ann Thorac Surg 68 : 235-237 (1999)

3) Nonaka M, Kadokura M, Tanio N, Yamamoto S, Kataoka D, Inoue K, Takaba T, Noguchi H and Nakajima H : Middle lobe bronchial obstruction after right upper lobectomy. J Bronchol 4 : 307-309 (1997)

4) Nonaka M, Kadokura M, Tanio N, Yamamoto S, Kataoka D and Takaba T: Bronchofiberscopic analysis for middle lobe bronchial deformity associated with right upper lobectomy. J Jpn Assoc Chest Surg 12: 474-481 (1998) (in Japanese)

5) Breiman RS, Beck JW, Korobkin M, Glency R, Akwari OE, Heaston DK, Moore AV and Ram PC: Volume determinations using computed tomography. AJR $138: 329-333$ (1982)

6) Frija J, Schmit P, Katz M, Vadrot $D$ and Laval-Jeantet $\mathbf{M}$ : Computed tomography of the pulmonary fissures : normal anatomy. J Comp Assist Tomogr 6 : 1069-1074 (1982)

7) Friedman PJ, Brimm JE, Botkin MC and Peters RM: Measuring lung volumes from chest films using equations derived from computed tomography. Invest Radiol $19: 263-268$ (1984)

8) Barnhard HJ, Pierce JA, Joyce JW and Bates JH : Roentgenographic determination of total lung capacity. Am J Med 28 : 51-60 (1960)

9) Irani B, Miller JE, Linberg E and Attar S : Use of radiopaque marking on middle lobe (or lingula) following upper resectional surgery. Ann Thorac Surg $5: 1-7$ (1968)

10) Wallace JE and Pillman R: Bronchographic studies after resection for pulmonary tuberculosis. Thorax 11 : 149-159 (1956)

11) Nonaka M, Kadokura M, Tanio N, Yamamoto S, Kataoka D, Inoue K and Takaba T: Changes in lung lobar volume and bronchial deformity after right upper lobectomy. Surg Today 28 : 285-288 (1998) 
12) Nonaka M, Kadokura M, Michihata T, Inoue $T$ and Takaba $T$ : How different surgical methods of performing right upper lobectomy contribute to postoperative bronchial branching deformity: an experimental study. Surg Today 29 : 610-613 (1999)

[Received August 8, 2000 : Accepted November 20, 2000] 